\title{
Conductance fluctuations in a disordered double-barrier junction
}

\author{
J. A. Melsen and C. W. J. Beenakker \\ Instituut-Lorentz, University of Leiden, P.O. Box 9506, 2300 RA Leiden, The Netherlands
}

(Received 16 January 1995)

\begin{abstract}
We consider the effect of disorder on coherent tunneling through two barriers in series, in the regime of overlapping transmission resonances. We present analytical calculations (using randommatrix theory) and numerical simulations (on a lattice) to show that strong mode mixing in the interbarrier region induces mesoscopic fluctuations in the conductance $G$ of universal magnitude $e^{2} / h$ for a symmetric junction. For an asymmetric junction, the root-mean-square fluctuations depend on the ratio $\nu$ of the two tunnel resistances according to $\operatorname{rms} G=\left(4 e^{2} / h\right) \beta^{-1 / 2} \nu(1+\nu)^{-2}$, where $\beta=1$ (2) in the presence (absence) of time-reversal symmetry.
\end{abstract}

\section{INTRODUCTION}

Resonant tunneling through two planar barriers in series is a textbook problem in quantum mechanics. Because of the separation of longitudinal and transverse motion, the problem is essentially one-dimensional and can be solved in an elementary way. Realistic double-barrier junctions contain in general some amount of disorder in the region between the barriers. At low temperatures and small applied voltages, the inelastic electron-phonon and electron-electron scattering processes are suppressed, but the elastic scattering by impurities remains. Scattering events couple the transverse and longitudinal motion of the tunneling electron, which substantially complicates the problem but also leads to additional physical effects.

The effects of disorder have been studied in the past ${ }^{1-4}$ with an emphasis on isolated transmission resonances (energy spacing between the resonances much greater than their width). Those studies are relevant for tunneling through a semiconductor quantum well, where the resonances are widely separated because the barrier separation $L$ is comparable to the Fermi wavelength $\lambda_{F}$. In the present paper we consider the opposite regime $L \gg \lambda_{F}$ of strongly overlapping resonances, relevant to metal structures (where $\lambda_{F}$ is very short, comparable to the interatomic separation), or to tunneling in the plane of a two-dimensional electron gas (where $L$ can be quite long, because of the large phase-coherence length). Two types of disorder can play a role, interface roughness at the barriers and impurities between the barriers. Interface roughness leads to mesoscopic (sample-to-sample) fluctuations in the conductance even in the absence of any phase coherence, because the tunnel probability $\Gamma$ of a single barrier depends strongly on its thickness. Conductance fluctuations for a single rough tunnel barrier have been studied by Raikh and Ruzin. ${ }^{5}$ Here we consider the case of impurity scattering in the absence of interface roughness. Phase coherence is then essential.

A methodological difference with earlier work on resonant tunneling is our use of random-matrix theory to describe the mode mixing in the interbarrier region. We assume that the disorder is weak enough that its effect on the average conductance is negligibly small. This requires a mean free path $l \gg \Gamma L$. Still, the disorder should be sufficiently strong to fully mix the transverse modes in the interbarrier region. This requires both $l \ll L / \Gamma$ and $W \ll L / \Gamma$ (where $W$ is the transverse dimension of the junction). We may then describe the disorder-induced mode mixing by a random $N \times N$ unitary matrix $(N$ being the total number of propagating transverse modes at the Fermi energy). This single assumption permits a complete analytical solution of the statistical properties of the conductance, using basic results for the so-called circular ensemble of random matrices. ${ }^{6}$ The circular ensemble is fully characterized by the symmetry index $\beta$, which equals $I$ in the presence of time-reversal symmetry (circular orthogonal ensemble) and 2 if time-reversal symmetry is broken by a magnetic field (circular unitary ensemble). (A third possibility, $\beta=4$, applies to zero magnetic field in the presence of strong spin-orbit scattering.)

As described in Sec. II, we find that the conductance $G$ of the double-barrier junction exhibits sample-to-sample fluctuations around the classical series conductance

$$
G_{\text {series }}=\left(2 e^{2} / h\right) N\left(1 / \Gamma_{1}+1 / \Gamma_{2}\right)^{-1} .
$$

(We denote by $\Gamma_{1}$ and $\Gamma_{2}$ the transmission probabilities per mode through barrier 1 and 2, and assume that these are mode independent and $\ll 1$.) We find that the rootmean-square fluctuations rms $G$ of the conductance depend only on the ratio $\nu=\Gamma_{1} / \Gamma_{2}$ of the two transmission probabilities, according to

$$
\operatorname{rms} G=\frac{4 e^{2}}{h} \beta^{-1 / 2} \frac{\nu}{(1+\nu)^{2}} .
$$

Corrections to Eq. (1.2) are smaller by a factor $e^{2} / h G_{\text {series }}$, which is $\ll 1$ if $N \Gamma_{i} \gg 1$. For a symmetric junction $(\nu=1)$ the fluctuations are of order $e^{2} / h$, independent of $N$ or $\Gamma_{i}$ (as long as $N \Gamma_{i} \gg 1$ ). This universality is reminiscent of the universal conductance fluctuations in diffusive metals. ${ }^{7,8}$ Just as in those systems, we expect the sample-to-sample fluctuations to be 
observable in a single sample, as reproducible fluctuations of the conductance as a function of Fermi energy or magnetic field.

Equation (1.2) assumes weak disorder, $l \gg \Gamma_{i} L$ (but still $\left.l \ll L / \Gamma_{i}\right)$. We generalize our results in Sec. III to stronger disorder, when the effects of the impurities on the average conductance have to be taken into account. As in a previous paper, ${ }^{9}$ where we considered a point-contact geometry, we do this by means of the Dorokhov-Mello-Pereyra-Kumar (DMPK) equation. ${ }^{10,11}$ We find that impurity scattering leads to the appearance of a weak-localization effect on the average conductance (observable as a negative magnetoresistance). The conductance fluctuations become independent of $\Gamma_{1}$ and $\Gamma_{2}$ if $L \gg l\left(\Gamma_{1}^{-1}+\Gamma_{2}^{-1}\right)$. A similar conclusion was reached previously by Iida, Weidenmüller, and Zuk, ${ }^{12}$ who studied the conductance fluctuations of a chain of disordered grains as a function of the coupling strength to two electron reservoirs. These authors found that the universal conductance fluctuations are recovered for a chain length $L$ much greater than some length $L_{0}$, which is parametrically greater than the mean free path. A more detailed comparison with Ref. 12 is not possible, because we consider a homogeneously disordered conductor rather than a chain of disordered grains.

To test our random-matrix description of mode mixing by weak disorder, we present in Sec. IV results from a numerical simulation of a disordered double-barrier junction defined on a two-dimensional lattice. The agreement with the theory is quite reasonable.

Two appendixes to the paper contain some technical material, which we need in the main text: In Appendix $A$, we present the analogue of the Dyson-Mehta formula ${ }^{13}$ for the circular ensemble, which expresses the variance of the conductance as a Fourier series. In Appendix B, we discuss the application to our problem of the method of moments ${ }^{14,15}$ for the DMPK equation.

\section{DOUBLE-BARRIER JUNCTION WITH STRONG MODE MIXING}

The double-barrier junction considered is shown schematically in the inset of Fig. 1. Since we assume $\lambda_{F} \ll L$, the scattering matrix $S$ of the whole system can be constructed from the scattering matrices $S_{i}$ of the individual barriers. The $2 N \times 2 N$ unitary matrix $S_{i}$ contains two $N \times N$ submatrices $r_{i}$ and $r_{i}^{\prime}$ (reflection from left to left and from right to right) and two other $N \times N$ submatrices $t_{i}$ and $t_{i}^{\prime}$ (transmission from left to right and from right to left). We use the polar decomposition ${ }^{16,17}$

$$
\begin{aligned}
S_{i}= & \left(\begin{array}{cc}
r_{i} & t_{i}^{\prime} \\
t_{i} & r_{i}^{\prime}
\end{array}\right) \\
= & \left(\begin{array}{cc}
U_{i} & 0 \\
0 & V_{i}
\end{array}\right)\left(\begin{array}{cc}
-i\left(1-\Gamma_{i}\right)^{1 / 2} & \Gamma_{i}^{1 / 2} \\
\Gamma_{i}^{1 / 2} & -i\left(1-\Gamma_{i}\right)^{1 / 2}
\end{array}\right) \\
& \times\left(\begin{array}{cc}
U_{i}^{\prime} & 0 \\
0 & V_{i}^{\prime}
\end{array}\right),
\end{aligned}
$$

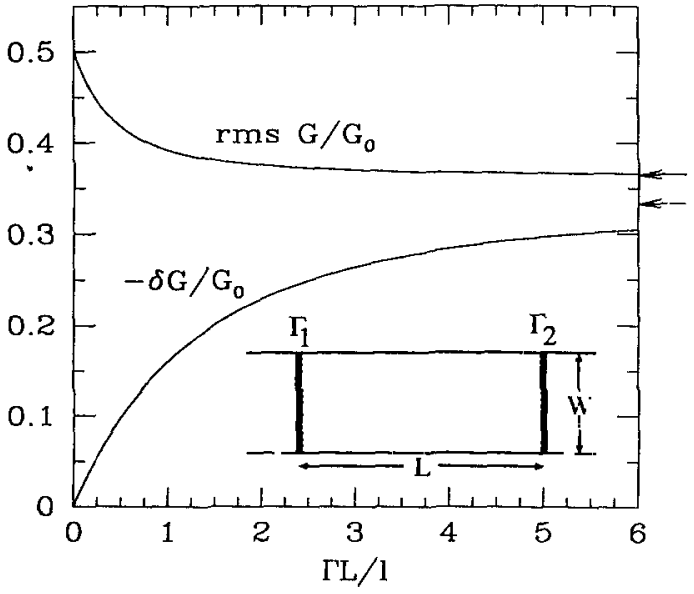

FIG. 1. Weak-localization correction $\delta G$ to the average conductance (in units of $G_{0}=2 e^{2} / h$ ) and root-mean-square fluctuations $\operatorname{rms} G \equiv(\operatorname{Var} G)^{1 / 2}$, computed from Eqs. (3.6) and (3.7) for $\beta=1$. The arrows give the limit $\Gamma L / l \gg 1$. The inset shows the geometry of the double-barrier junction (the disordered region is dotted). The curves plotted in the figure are for a symmetric junction, $\Gamma_{1}=\Gamma_{2} \equiv \Gamma \ll 1$.

where the $U$ 's and $V$ 's are $N \times N$ unitary matrices. In zero magnetic field, $U_{i}^{\prime}=U_{i}^{T}$ and $V_{i}^{\prime}=V_{i}^{T}$, so that $S_{i}$ is symmetric - as it should be in the presence of time-reversal symmetry. The transmission matrix $t$ of the whole system is given by

$$
t=t_{2}\left(1-r_{1}^{\prime} r_{2}\right)^{-1} t_{1} .
$$

Substitution of the polar decomposition (2.1) yields the matrix product $t t^{\dagger}$ in the form

$$
\begin{aligned}
t t^{\dagger} & =V_{2}\left[a+\frac{1}{2} b\left(\Omega+\Omega^{\dagger}\right)\right]^{-1} V_{2}^{\dagger}, \\
\Omega & =U_{2}^{\prime} V_{1} V_{1}^{\prime} U_{2}, \\
a & =\left[1+\left(1-\Gamma_{1}\right)\left(1-\Gamma_{2}\right)\right] / \Gamma_{1} \Gamma_{2}, \\
b & =2 \sqrt{\left(1-\Gamma_{1}\right)\left(1-\Gamma_{2}\right)} / \Gamma_{1} \Gamma_{2} .
\end{aligned}
$$

The eigenvalues $T_{n}$ of $t t^{\dagger}$ are related to the eigenvalues $\exp \left(i \phi_{n}\right)$ of $\Omega$ by

$$
T_{n}=\left(a+b \cos \phi_{n}\right)^{-1} .
$$

The $T_{n}$ 's determine the conductance $G$ of the doublebarrier junction, according to the Landauer formula

$$
G=G_{0} \sum_{n=1}^{N} T_{n}
$$

where $G_{0}=2 e^{2} / h$ is the conductance quantum.

We consider an isotropic ensemble of double-barrier junctions, analogous to the isotropic ensemble of disordered wires. ${ }^{16}$ We assume that $l \ll L / \Gamma_{i}$ and $W \ll L / \Gamma_{i}$, so that the tunneling is accompanied by strong mode mixing: An electron entering the junction in mode $n$ is randomly distributed among all modes $m$ before leaving the junction. We assume in this section that mode 
mixing is the dominant effect of the disorder, and that the reduction of the average conductance by the impurity scattering can be neglected. This requires $l \gg \Gamma_{i} L$. (The case of stronger disorder is treated in the next section.) In the polar decomposition (2.1), the mode mixing is accounted for by the unitary matrices $U$ and $V$. The number of different unitary matrices is $2 \beta$, where $\beta=1$ in zero magnetic field and $\beta=2$ if time-reversal symmetry is broken by a magnetic field. The isotropic ensemble is the ensemble where the $2 \beta$ unitary matrices are independently and uniformly distributed over the unitary group. In other words, the $U$ 's and $V$ 's are drawn independently from the circular unitary ensemble (CUE) of random-matrix theory. ${ }^{6}$

To determine the statistics of the conductance (2.5) we need the probability distribution $P\left(\left\{\phi_{n}\right\}\right)$ of the eigenvalues of $\Omega$. For $\beta=2, \Omega=U_{2}^{\prime} V_{1} V_{1}^{\prime} U_{2}$ is the product of four independent matrices from the CUE, and hence $\Omega$ is also distributed according to the CUE. For $\beta=1$, $\Omega=U_{2}^{T} V_{1} V_{1}^{T} U_{2}$ is of the form $W W^{T}$ with $W$ a member of the CUE. The ensemble of $\Omega$ is then the circular orthogonal ensemble (COE). The distribution of the eigenvalues in the CUE and COE is given by ${ }^{6}$

$$
P\left(\left\{\phi_{n}\right\}\right)=C \prod_{n<m}\left|\exp \left(i \phi_{n}\right)-\exp \left(i \phi_{m}\right)\right|^{\beta},
$$

where $C$ is a normalization constant.

We compute the average $\langle A\rangle$ and variance $\operatorname{Var} A=$ $\left\langle A^{2}\right\rangle-\langle A\rangle^{2}$ of linear statistics $A=\sum_{n=1}^{N} a\left(\phi_{n}\right)$ on the eigenphases $\phi_{n}$. Since in the circular ensemble the $\phi_{n}$ 's are uniformly distributed in $(0,2 \pi)$, the average is exactly equal to

$$
\langle A\rangle=\frac{N}{2 \pi} \int_{0}^{2 \pi} d \phi a(\phi) .
$$

An exact expression for the variance can also be given, ${ }^{6}$ but is cumbersome to evaluate. For $N \gg 1$, we can use a variation on the Dyson-Mehta formula ${ }^{13}$ (derived in Appendix A), ${ }^{18}$

$$
\begin{aligned}
\operatorname{Var} A & =\frac{1}{\pi^{2} \beta} \sum_{n=1}^{\infty} n\left|a_{n}\right|^{2}+O\left(N^{-1}\right) \\
a_{n} & =\int_{0}^{2 \pi} d \phi \mathrm{e}^{i n \phi} a(\phi) .
\end{aligned}
$$

For the conductance [given by Eqs. (2.4) and (2.5)], we substitute $a(\phi)=(a+b \cos \phi)^{-1}$, with Fourier coefficients $a_{n}=2 \pi\left(a^{2}-b^{2}\right)^{-1 / 2} b^{-n}\left[\left(a^{2}-b^{2}\right)^{1 / 2}-a\right]^{n}$. The results are

$$
\begin{aligned}
\left\langle G / G_{0}\right\rangle & =N\left(1 / \Gamma_{1}+1 / \Gamma_{2}-1\right)^{-1}, \\
\operatorname{Var} G / G_{0} & =\frac{4}{\beta} \frac{\left(1-\Gamma_{1}\right)\left(1-\Gamma_{2}\right) \Gamma_{1}^{2} \Gamma_{2}^{2}}{\left(\Gamma_{1}+\Gamma_{2}-\Gamma_{1} \Gamma_{2}\right)^{4}} .
\end{aligned}
$$

Equation (2.9) for the average conductance is what one would expect from classical addition of the resistances $\left(N \Gamma_{i} G_{0}\right)^{-1}$ of the individual barriers. [The -1 in Eq. (2.9) corrects for a double counting of the contact resistance and becomes irrelevant for $\Gamma_{i} \ll 1$.] Each member of the ensemble contains a different set of overlapping transmission resonances, and the ensemble average removes any trace of resonant tunneling in $\langle G\rangle$. In a previous paper, ${ }^{19}$ we have shown that the average conductance differs drastically from the series conductance if the double-barrier junction is connected to a superconductor, but here we consider only normal-metal conductors.

Equation (2.10) for the conductance fluctuations tells us that $\operatorname{Var} G$ becomes completely independent of $N$ in the limit $N \rightarrow \infty$. [More precisely, corrections to Eq. (2.10) are of order $\left\langle G / G_{0}\right\rangle^{-1}$, which is $\ll 1$ if $N \Gamma_{i} \gg 1$.] Since $\Gamma_{i} \ll 1$, we may simplify Eq. (2.10) to

$$
\operatorname{Var} G / G_{0}=\frac{4}{\beta} \frac{\Gamma_{1}^{2} \Gamma_{2}^{2}}{\left(\Gamma_{1}+\Gamma_{2}\right)^{4}}
$$

which depends only on the ratio $\Gamma_{1} / \Gamma_{2}$ and not on the individual $\Gamma_{i}$ 's. The variance reaches a $\Gamma$-independent maximum for two equal barriers,

$$
\operatorname{Var} G / G_{0}=\frac{1}{4} \beta^{-1} \quad \text { if } \Gamma_{1}=\Gamma_{2} .
$$

The variance is almost twice the result $\frac{2}{15} \beta^{-1}$ for an isotropic ensemble of disordered wires, ${ }^{14,15}$ and precisely twice the result $\frac{1}{8} \beta^{-1}$ for an isotropic ensemble of ballistic quantum dots. ${ }^{12,20,21}$

\section{EFFECTS OF STRONG DISOR.DER}

In this section we relax the assumption $l \gg \Gamma_{i} L$ of Sec. II, to include the case that the impurity scattering is sufficiently strong to affect the average conductance. We assume $W \ll L$, so that we are justified in using an isotropic distribution for the scattering matrix $S_{L}$ of the interbarrier region. ${ }^{16}$ The scattering matrix $S$ of the entire system is now composed from the three scattering matrices $S_{1}, S_{L}$, and $S_{2}$ in series. The composition is most easily carried out in terms of the transfer matrices $M_{1}, M_{L}$, and $M_{2}$ associated with $S_{1}, S_{L}$, and $S_{2}$, respectively. The transfer matrix $M$ of the entire system is the matrix product $M=M_{2} M_{L} M_{1}$, so the total distribution $P(M)$ is a convolution of the individual distributions $P_{1}\left(M_{1}\right), P_{L}\left(M_{L}\right)$, and $P_{2}\left(M_{2}\right): P=P_{2} \circ P_{L} \circ P_{1}$, where the convolution $\circ$ is defined by

$$
P_{i} \circ P_{j}(M)=\int d M^{\prime} P_{i}\left(M M^{\prime-1}\right) P_{j}\left(M^{\prime}\right) .
$$

The isotropy assumption implies that each distribution $P_{i}\left(M_{i}\right)$ is only a function of the eigenvalues of $M_{i} M_{i}^{\dagger}$.

We now use the fact that the convolution of isotropic distributions of transfer matrices commutes. (A proof is given in Ref. 9.) This permits us to consider an equivalent system, with transfer matrix $M=M_{L} M_{2} M_{1}$, where all disorder is at one side of the double-barrier junction instead of in between the barriers. The $L$ dependence of the distribution of transmission eigenvalues for this system is governed by the DMPK equation, ${ }^{10,11}$ 


$$
\begin{aligned}
\frac{\partial}{\partial s} P\left(\left\{\lambda_{n}\right\}, s\right)= & \frac{2}{\beta N+2-\beta} \\
& \times \sum_{i=1}^{N} \frac{\partial}{\partial \lambda_{2}}\left(\lambda_{2}\left(1+\lambda_{2}\right) J \frac{\partial}{\partial \lambda_{2}} \frac{P}{J}\right), \\
J= & \prod_{\imath<j}\left|\lambda_{2}-\lambda_{\jmath}\right|^{\beta},
\end{aligned}
$$

where $s=L / l$ and $\lambda_{n}=\left(1-T_{n}\right) / T_{n}$. The initial condition $(s \rightarrow 0)$ of Eq. (3.2) corresponds to taking $M_{L}=1$, which implies for $P$ the isotropic ensemble given by Eq. (2.6).

To compute the $L$ dependence of the mean and variance of the conductance, we use the method of moments of Mello ${ }^{14}$ and Mello and Stone, ${ }^{15}$ who have derived a hierarchy of differential equations for the moments of $\mathcal{T}_{q} \equiv \sum_{n=1}^{N} T_{n}^{q}$. The hierarchy closes order by order in an expansion in powers of $1 / N$. Mello and Stone considered a ballistic initial condition, corresponding to $\left\langle\mathcal{T}_{q}^{p}\right\rangle \rightarrow N^{p}$ for $s \rightarrow 0$. We have the different initial condition of a double-barrier junction. The differential equations and initial conditions for the moments are given in Appendix $B$. For the mean conductance and its variance we obtain

$$
\begin{aligned}
\left\langle G / G_{0}\right\rangle= & \frac{N}{s+\rho}+\frac{1}{3}(1-2 / \beta) \\
& -\frac{1-2 / \beta}{(s+\rho)^{3}}\left[s^{2}-s\left(a-\rho-\rho^{2}\right)+\frac{1}{3} \rho^{3}\right],
\end{aligned}
$$

$$
\begin{aligned}
\operatorname{Var} G / G_{0}= & \frac{2}{15 \beta}+\frac{2}{\beta(s+\rho)^{6}}\left[s ^ { 2 } \left(\frac{1}{2} a^{2}+\frac{1}{2} \rho^{2}-2 a \rho^{2}\right.\right. \\
& \left.+\rho^{4}\right)+s\left(-2 a^{2} \rho+2 a \rho^{3}-\frac{2}{5} \rho^{5}\right) \\
& \left.+\frac{1}{2} a^{2} \rho^{2}-\frac{1}{2} \rho^{4}-\frac{1}{15} \rho^{6}\right]
\end{aligned}
$$

where $a$ has been defined in Eq. (2.3) and $\rho$ is defined by

$$
\rho=1 / \Gamma_{1}+1 / \Gamma_{2}-1 .
$$

Corrections to Eqs. (3.3) and (3.4) are of order $(s+$ $\rho) / N$. For two equal barriers $\left(\Gamma_{1}=\Gamma_{2} \equiv \Gamma\right)$ in the limit $\Gamma \rightarrow 0$ at fixed $\Gamma s$, Eqs. (3.3) and (3.4) simplify to

$$
\begin{aligned}
\delta G / G_{0} & \equiv\left\langle G / G_{0}\right\rangle-N(s+\rho)^{-1} \\
& =\frac{1}{3}(1-2 / \beta)-\frac{1-2 / \beta}{(2+\Gamma s)^{3}}\left(\frac{8}{3}+2 \Gamma s\right), \\
\operatorname{Var} G / G_{0} & =\frac{2}{15 \beta}+\frac{4}{\beta(2+\Gamma s)^{6}}\left(\Gamma^{2} s^{2}+\frac{8}{5} \Gamma s+\frac{28}{15}\right) .
\end{aligned}
$$

Equations (3.6) and (3.7) are plotted in Fig. 1 (for $\beta=$ $1)$. In the limit of large disorder $(\Gamma s \gg 1)$, we recover the familiar results ${ }^{14,15}$ for a disordered wire: $\delta G / G_{0}=$ $\frac{1}{3}(1-2 / \beta), \operatorname{Var} G / G_{0}=\frac{2}{15} \beta^{-1}$ (indicated by arrows in Fig. 1). In the opposite limit $\Gamma s \ll 1$, we find $\delta G=0$, $\operatorname{Var} G / G_{0}=\frac{1}{4} \beta^{-1}-$ as in Sec. II [cf. Eqs. (2.9) and (2.12)].

\section{NUMERICAL SIMULATIONS}

To test our results we have performed numerical simulations, using the recursive Green's function method of Ref. 22. The disordered interbarrier region was modeled by a tight-binding Hamiltonian on a two-dimensional square lattice with lattice constant $d$. The Fermi energy was chosen at $1.5 u_{0}$ from the band bottom, with $u_{0}=\hbar^{2} / 2 m d^{2}$. Disorder was introduced by randomly assigning a value between $\pm \frac{1}{2} U_{D}$ to the on-site potential of the lattice points in a rectangle with $L=142 d, W=71 d$ (corresponding to $N=30$ ). We chose $U_{D}=0.6 u_{0}$, corresponding to $L / l=0.9$. The transfer matrix $M_{L}$ was computed numerically, and then multiplied with the transfer matrices $M_{1}$ and $M_{2}$ of the two barriers (which we constructed analytically, given the mode-independent tunnel probabilities $\Gamma_{1}$ and $\Gamma_{2}$ ). We took $\Gamma_{2}=0.15$ and varied $\Gamma_{1}$ between 0.05 and 0.5 . These parameter values were chosen in order to be close to the regime $\Gamma_{\imath} L \ll l \ll L / \Gamma_{\imath}, W \ll L / \Gamma_{\imath}$ in which disorder is expected to cause strong mode mixing, without having a large effect on the average conductance (the regime studied in Sec. II).

In Fig. 2 we show the comparison between theory and simulation. The solid curve is $\operatorname{Var} G / G_{0}$ computed from 2250 realizations of the disorder potential. The dotted curve is the theoretical prediction from Eq. (3.4) for the parameter values of the simulation (and for $\beta=1$, since there was no magnetic field). There are no adjustable parameters. The agreement is quite reasonable. It is likely that the remaining discrepancy is due to the fact that the theoretical condition $N \Gamma_{2} \gg 1$ was not well met in the simulation (where $N \Gamma_{2}=4.5$ ). The value $N=30$ of the simulation is already at the limit of our computational capabilities and we are not able to provide a more stringent numerical test of the theory.

Note added in proof: The case $\Gamma_{1}=\Gamma_{2} \ll 1 / s$ has recently been considered by V. I. Fal'ko [Phys. Rev. B 51, 5227 (1995)]. This result differes from our Eq. (2.12) in the numerical coefficient.

\section{ACKNOWLEDGMENTS}

Discussions with P. W. Brouwer have been most helpful. This research was supported by the "Nederlandse

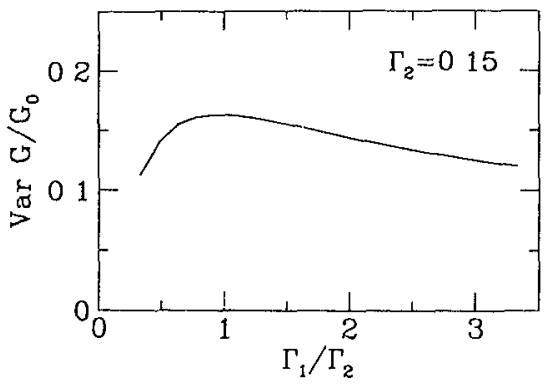

FIG. 2. Solid curve: variance of the conductance from a numerical simulation of an ensemble of disordered double-barrier junctions $(L / W=2, N=30, s=0.9)$, as a function of the ratio $\Gamma_{1} / \Gamma_{2}$, with $\Gamma_{2}=0.15$ held constant. There is no magnetic field $(\beta=1)$. The dashed curve is the prediction from Eq. (3.4). There are no adjustable parameters. 
organisatie voor Wetenschappelijk Onderzoek" (NWO) and by the "Stichting voor Fundamenteel Onderzoek der Materie" (FOM).

\section{APPENDIX A: DYSON-MEHTA FORMULA FOR THE CIRCULAR ENSEMBLE}

The variance $\operatorname{Var} A$ of a linear statistic $A=$ $\sum_{n=1}^{N} a\left(\phi_{n}\right)$ on the eigenphases is given by a double integral,

$$
\operatorname{Var} A=-\int_{0}^{2 \pi} d \phi \int_{0}^{2 \pi} d \phi^{\prime} a(\phi) a\left(\phi^{\prime}\right) K\left(\phi, \phi^{\prime}\right)
$$

over the two-point correlation function

$$
K\left(\phi, \phi^{\prime}\right)=\langle\rho(\phi)\rangle\left\langle\rho\left(\phi^{\prime}\right)\right\rangle-\left\langle\rho(\phi) \rho\left(\phi^{\prime}\right)\right\rangle .
$$

The brackets $\langle\cdots\rangle$ denote an average over the circular ensemble, and

$$
\rho(\phi)=\sum_{n=1}^{N} \delta\left(\phi-\phi_{n}\right)
$$

is the microscopic density of eigenphases. In this appendix we compute $K\left(\phi, \phi^{\prime}\right)$ in the large- $N$ limit, using the method of functional derivatives of Ref. 23. This leads to Eq. (2.8) for $\operatorname{Var} A$, which is the analogue for the circular ensemble of the Dyson-Mehta formula for the Gaussian ensemble. ${ }^{13}$ The analogy is straightforward, but we have not found it in the literature. ${ }^{18}$

We consider a generalized circular ensemble, with probability distribution

$$
\begin{aligned}
P_{V}\left(\left\{\phi_{n}\right\}\right) & =C \exp \left[-\beta\left(\sum_{i<j} U\left(\phi_{i}-\phi_{j}\right)+\sum_{i=1}^{N} V\left(\phi_{i}\right)\right)\right] \\
C^{-1} & =\int_{0}^{2 \pi} d \phi_{1} \int_{0}^{2 \pi} d \phi_{2} \cdots \int_{0}^{2 \pi} d \phi_{N} P_{V}\left(\left\{\phi_{n}\right\}\right) \\
U(\phi) & =-\ln \left|2 \sin \frac{1}{2} \phi\right|
\end{aligned}
$$

The "potential" $V(\phi)$ is arbitrary. If $V \equiv \mathbf{0}, \mathrm{Eq}$. (A4) is the same as the distribution (2.6) of the circular ensemble. The brackets $\langle\cdots\rangle_{V}$ denote an average with the $V$-dependent distribution (A4). Following Ref. 23, we express the two-point correlation function as a functional derivative of the density with respect to the potential,

$$
K\left(\phi, \phi^{\prime}\right)=\frac{1}{\beta} \frac{\delta\langle\rho(\phi)\rangle_{V}}{\delta V\left(\phi^{\prime}\right)} .
$$

The functional derivative can be computed in the large- $N$ limit from the relationship ${ }^{24}$

$$
-\int_{0}^{2 \pi} d \phi^{\prime} U\left(\phi-\phi^{\prime}\right)\left\langle\rho\left(\phi^{\prime}\right)\right\rangle_{V}=V(\phi)+\text { const. }
$$

Corrections to Eq. (A6) are smaller by a factor $1 / N$. The additive constant is obtained from the normalization $\int d \phi\langle\rho(\phi)\rangle_{V}=N$.
Fourier transformation of Eq. (A6) yields

$$
-\frac{\pi}{|n|}\left\langle\rho_{n}\right\rangle_{V}=V_{n}, \quad n \neq 0 .
$$

We have defined the Fourier coefficients

$$
f_{n}=\int_{0}^{2 \pi} d \phi \mathrm{e}^{i n \phi} f(\phi)
$$

and we have used that $U_{n}=\pi /|n|$ for $n \neq 0$. From Eqs. (A5) and (A6), we see that $K\left(\phi, \phi^{\prime}\right)=K\left(\phi-\phi^{\prime}\right)$ depends on the difference $\phi-\phi^{\prime}$ only, and is independent of $V$. The Fourier coefficients of $K(\phi)$ are

$$
K_{n}=-|n| / \pi \beta
$$

for $n \neq 0$. Since $K_{0}=0$ by definition, Eq. (A9a) holds in fact for all $n$. Inversion of the Fourier transform yields the correlation function

$$
K(\phi)=-\frac{1}{\pi^{2} \beta} \frac{d^{2}}{d \phi^{2}} \ln \left|\sin \frac{1}{2} \phi\right|,
$$

which has an integrable singularity at $\phi=0$. For $\phi \neq 0$, $K(\phi)=\left[4 \pi^{2} \beta \sin ^{2}(\phi / 2)\right]^{-1}$. Substitution of Eq. (A9) into Eq. (A1) gives the required analogue of the DysonMehta formula for the large- $N$ limit of the variance of a linear statistic,

$$
\begin{aligned}
\operatorname{Var} A= & -\frac{1}{\pi^{2} \beta} \int_{0}^{2 \pi} d \phi \int_{0}^{2 \pi} d \phi^{\prime}\left(\frac{d a(\phi)}{d \phi}\right) \\
& \times\left(\frac{d a\left(\phi^{\prime}\right)}{d \phi^{\prime}}\right) \ln \left|\sin \frac{\phi-\phi^{\prime}}{2}\right| \\
= & \frac{1}{\pi^{2} \beta} \sum_{n=1}^{\infty} n\left|a_{n}\right|^{2} .
\end{aligned}
$$

\section{APPENDIX B: MOMENT EXPANSION OF THE DMPK EQUATION}

Mello and Stone ${ }^{15}$ have derived from the DMPK equation (3.2) a hierarchy of differential equations for the moments of $\mathcal{T}_{q}=\sum_{n=1}^{N} T_{n}^{q}$. The hierarchy closes order by order in the series expansion

$$
\begin{aligned}
\left\langle\mathcal{T}^{p}\right\rangle= & N^{p} f_{p, 0}(s)+N^{p-1} f_{p, 1}(s) \\
& +N^{p-2} f_{p, 2}(s)+\cdots \\
\left\langle\mathcal{T}^{p} \mathcal{T}_{2}\right\rangle= & N^{p+1} g_{p+1,0}(s)+N^{p} g_{p+1,1}(s) \\
& +N^{p-1} g_{p+1,2}(s)+\cdots \\
\left\langle\mathcal{T}^{p} \mathcal{T}_{3}\right\rangle= & N^{p+1} h_{p+1,0}(s)+N^{p} h_{p+1,1}(s) \\
& +N^{p-1} h_{p+1,2}(s)+\cdots, \\
\left\langle\mathcal{T}^{p} \mathcal{T}_{2}^{2}\right\rangle= & N^{p+2} l_{p+2,0}(s)+N^{p+1} l_{p+2,1}(s) \\
& +N^{p} l_{p+2,2}(s)+\cdots,
\end{aligned}
$$

where we have defined $\mathcal{T} \equiv \mathcal{T}_{1}$. For a calculation of $\operatorname{Var} G$ we need to determine $\left\langle\mathcal{T}^{p}\right\rangle$ down to $O\left(N^{p-2}\right),\left\langle\mathcal{T}^{p} \mathcal{T}_{2}\right\rangle$ down to $O\left(N^{p}\right)$, and $\left\langle\mathcal{T}^{p} \mathcal{T}_{3}\right\rangle$ and $\left\langle\mathcal{T}^{p} \mathcal{T}_{2}^{2}\right\rangle$ only to the highest occurring order. The resulting set of differential equations we have to solve is ${ }^{15}$ 


$$
\begin{gathered}
f_{p, 0}^{\prime}(s)+p f_{p+1,0}(s)=0, \\
g_{p, 0}^{\prime}(s)+(p+3) g_{p+1,0}(s)=2 f_{p+1,0}(s), \\
f_{p, 1}^{\prime}(s)+p f_{p+1,1}(s)=(1-2 / \beta)\left[f_{p, 0}^{\prime}(s)+p g_{p, 0}(s)\right] \\
l_{p, 0}^{\prime}(s)+(p+6) l_{p+1,0}(s)=4 g_{p+1,0}(s), \\
h_{p, 0}^{\prime}(s)+(p+5) h_{p+1,0}(s)=6 g_{p+1,0}(s)-3 l_{p+1,0}(s) \\
g_{p, 1}^{\prime}(s)+(p+3) g_{p+1,1}(s) \\
=2 f_{p+1,1}(s)-(1-2 / \beta)\left[-g_{p, 0}^{\prime}(s)+2 g_{p, 0}(s)\right. \\
\left.\quad-4 h_{p, 0}(s)-(p-1) l_{p, 0}(s)\right] \\
f_{p, 2}^{\prime}(s)+p f_{p+1,2}(s) \\
=(1-2 / \beta)\left[f_{p, 1}^{\prime}(s)+p g_{p, 1}(s)\right] \\
+2 \beta^{-1} p(p-1)\left[g_{p-1,0}(s)-h_{p-1,0}(s)\right] .
\end{gathered}
$$

We need to determine the initial conditions $f(0)$, $g(0), h(0)$, and $l(0)$ from the distribution function (2.6) for the eigenphases in the circular ensemble. In the large- $N$ limit, the linear statistic $\mathcal{T}_{q}$ on the eigenphases has a Gaussian distribution with a width of order $N^{0}$. Therefore, if we write $\mathcal{T}_{q}=\left\langle\mathcal{T}_{q}\right\rangle+\delta \mathcal{T}_{q}$, we know that $\left\langle\mathcal{T}_{q}\right\rangle=O(N),\left\langle\delta \mathcal{T}_{q}\right\rangle=0,\left\langle\left(\delta \mathcal{T}_{q}\right)^{2 n+1}\right\rangle=O\left(N^{-1}\right)$ and $\left\langle\left(\delta \mathcal{T}_{q}\right)^{2 n}\right\rangle=O\left(N^{0}\right)$. This implies that, for $s \rightarrow 0$,

$$
\begin{aligned}
\left\langle\mathcal{T}^{p}\right\rangle & =\langle\mathcal{T}\rangle^{p}+\frac{1}{2} p(p-1)\langle\mathcal{T}\rangle^{p-2}\left\langle(\delta \mathcal{T})^{2}\right\rangle+O\left(N^{p-4}\right), \\
\left\langle\mathcal{T}^{p} \mathcal{T}_{2}\right\rangle & =\langle\mathcal{T}\rangle^{p}\left\langle\mathcal{T}_{2}\right\rangle+O\left(N^{p-1}\right), \\
\left\langle\mathcal{T}^{p} \mathcal{T}_{3}\right\rangle & =\langle\mathcal{T}\rangle^{p}\left\langle\mathcal{T}_{3}\right\rangle+O\left(N^{p-1}\right),
\end{aligned}
$$

$$
\left\langle\mathcal{T}^{p} \mathcal{T}_{2}^{2}\right\rangle=\langle\mathcal{T}\rangle^{p}\left\langle\mathcal{T}_{2}\right\rangle^{2}+O\left(N^{p}\right)
$$

The average $\left\langle(\delta \mathcal{T})^{2}\right\rangle$ is just $\operatorname{Var} G / G_{0}$, which is given by Eq. (2.10),

$$
\left\langle(\delta \mathcal{O})^{2}\right\rangle=\beta^{-1} b^{2} p^{-4}+O\left(N^{-1}\right) .
$$

The other averages in Eq. (B3) follow from

$$
\left\langle\mathcal{T}_{q}\right\rangle=\frac{N}{2 \pi} \int_{0}^{2 \pi} d \phi(a+b \cos \phi)^{-q}
$$

The resulting initial conditions read

$$
\begin{aligned}
& f_{p, 0}(0)=\rho^{-p}, f_{p, 1}(0)=0 \\
& f_{p, 2}(0)=\frac{1}{2} \beta^{-1} p(p-1) \rho^{-(p+2)} b^{2} \\
& g_{p, 0}(0)=a \rho^{-(p+2)}, g_{p, 1}(0)=0 \\
& h_{p, 0}(0)=\rho^{-(p+2)}\left(\frac{3}{2} a^{2} \rho^{-2}-\frac{1}{2}\right) \\
& l_{p, 0}(0)=a^{2} \rho^{-(p+4)} .
\end{aligned}
$$

The set of differential equations (B2) can be solved by substitution of the following Ansatz for the $p$ dependence (adapted from Ref. 25):

$$
x_{p, l}(s)=(s+\rho)^{-(p+2 l+n)}\left[p^{2} \varphi(s)+p \chi(s)+\psi(s)\right],
$$

where $n=0$ if $x$ is $f, n=3$ if $x$ is $g$, and $n=6$ if $x$ is $h$ or $x$ is $l$. The mean and variance of the conductance, to order $N^{-1}$, then follow from

$$
\begin{aligned}
\left\langle G / G_{0}\right\rangle= & N f_{1,0}(s)+f_{1,1}(s), \\
\operatorname{Var} G / G_{0}= & N^{2}\left[f_{2,0}(s)-f_{1,0}(s)^{2}\right]+N\left[f_{2,1}(s)\right. \\
& \left.-2 f_{1,0}(s) f_{1,1}(s)\right]+f_{2,2}(s) \\
& -2 f_{1,0}(s) f_{1,2}(s)-f_{1,1}(s)^{2} .
\end{aligned}
$$

The results are Eqs. (3.3) and (3.4).
${ }^{1}$ H. A. Fertig and S. Das Sarma, Phys. Rev. B 40, 7410 (1989); H. A. Fertig, S. He, and S. Das Sarma, ibid. 41, 3596 (1990).

2 J. Leo and A. H. MacDonald, Phys. Rev. Lett. 64, 817 (1990).

${ }^{3}$ R. Berkovits and S. Feng, Phys. Rev. B 45, 97 (1992).

${ }^{4}$ I. V. Lerner and M. E. Raikh, Phys. Rev. B 45, 14036 (1992).

${ }^{5}$ M. E. Raikh and I. M. Ruzin, in Mesoscopic Phenomena in Solids, edited by B. L. Al'tshuler, P. A. Lee, and R. A. Webb (North-Holland, Amsterdam, 1991).

${ }^{6}$ M. L. Mehta, Random Matrices (Academic, New York, 1991).

${ }^{7}$ B. L. Al'tshuler, Pis'ma Zh. Eksp. Teor. Fiz. 41, 530 (1985) [JETP Lett. 41, 648 (1985)].

${ }^{8}$ P. A. Lee and A. D. Stone, Phys. Rev. Lett. 55, 1622 (1985).

${ }^{9}$ C. W. J. Beenakker and J. A. Melsen, Phys. Rev. B 50, 2450 (1994).
${ }^{10}$ O. N. Dorokhov, Pis'ma Zh. Eksp. Teor. Fiz. 36, 259 (1982) [JETP Lett. 36, 318 (1982)].

${ }^{11}$ P. A. Mello, P. Pereyra, and N. Kumar, Ann. Phys. (N. Y.) 181, 290 (1988).

${ }^{12}$ S. Iida, H. A. Weidenmüller, and J. A. Zuk, Phys. Rev. Lett. 64, 583 (1990); Ann. Phys. (N.Y.) 200, 219 (1990).

${ }^{13}$ F. J. Dyson and M. L. Mehta, J. Math. Phys. 4, 701 (1963).

${ }^{14}$ P. A. Mello, Phys. Rev. Lett. 60, 1089 (1988).

${ }^{15}$ P. A. Mello and A. D. Stone, Phys. Rev. B 44, 3559 (1991).

${ }^{16}$ A. D. Stone, P. A. Mello, K. A. Muttalib, and J.-L. Pichard, in Mesoscopic Phenomena in Solids (Ref. 5).

${ }^{17}$ Th. Martin and R. Landauer, Phys. Rev. B 45, 1742 (1992).

${ }^{18}$ A different derivation of Eq. (2.8) has been given recently by P. J. Forrester, Nucl. Phys. B 435, 421 (1995).

${ }^{19}$ J. A. Melsen and C. W. J. Beenakker, Physica B 203, 219 (1994).

${ }^{20}$ R. A. Jalabert, J.-L. Pichard, and C. W. J. Beenakker, Europhys. Lett. 27, 255 (1994). 
${ }^{21}$ H. U. Baranger and P. A. Mello, Phys. Rev. Lett. 73, 142 (1994).

${ }^{22}$ H. U. Baranger, D. P. DiVincenzo, R. A. Jalabert, and A. D. Stone, Phys. Rev. B 44, 10637 (1991). The computer code for the recursive Green's function calculation was kindly made available to us by Dr. Jalabert.
${ }^{23}$ C. W. J. Beenakker, Phys. Rev. Lett. 70, 1155 (1993); Phys. Rev. B 47, 15763 (1993).

${ }^{24}$ F. J. Dyson, J. Math. Phys. 13, 90 (1972).

${ }^{25}$ M. J. M. de Jong and C. W. J. Beenakker, Phys. Rev. B 46, 13400 (1992). 